Interfaces and Free Boundaries 15 (2013), 429-450

DOI 10.4171/IFB/309

\title{
On the role of kinetic and interfacial anisotropy in the crystal growth theory
}

\author{
MI-Ho GIGA \\ Graduate School of Mathematical Sciences, University of Tokyo, 3-8-1 Komaba Meguro-ku, Tokyo \\ 153-8914, Japan \\ E-mail:mihogiga@ms.u-tokyo.ac.jp \\ YOSHIKAZU GIGA \\ Graduate School of Mathematical Sciences, University of Tokyo, 3-8-1 Komaba Meguro-ku, Tokyo \\ 153-8914, Japan \\ E-mail: labgiga@ms.u-tokyo.ac.jp
}

[Received 28 September 2012 and in revised form 13 September 2013]

\begin{abstract}
A planar anisotropic curvature flow equation with constant driving force term is considered when the interfacial energy is crystalline. The driving force term is given so that a closed convex set grows if it is sufficiently large. If initial shape is convex, it is shown that a flat part called a facet (with admissible orientation) is instantaneously formed. Moreover, if the initial shape is convex and slightly bigger than the critical size, the shape becomes fully faceted in a finite time provided that the Frank diagram of interfacial energy density is a regular polygon centered at the origin. The proofs of these statements are based on approximation by crystalline algorithm whose foundation was established a decade ago. Our results indicate that the anisotropy of interfacial energy plays a key role when crystal is small in the theory of crystal growth. In particular, our theorems explain a reason why snow crystal forms a hexagonal prism when it is very small.
\end{abstract}

2010 Mathematics Subject Classification: Primary 35K67, 35B36, 35D40, 35 Q80.

Keywords: Facet; curvature flow equation; crystalline flow; viscosity solution

\section{Introduction}

In a growing crystal it often appears a flat surface called a facet. We are interested in explaining in what way such a facet is formed using a macroscopic model based on thermomechanics [38]. When a crystal is very small, it is sometimes observed that all surfaces of crystals are facets. Such a shape is often called fully faceted. In other words, it is a polytope. A typical example is a beautiful hexagonal prism of a snowflake. Formation of facets as well as fully faceted shapes is due to anisotropy of crystals since without anisotropy no facet is expected like fluid drops. There are at least two types of anisotropies - interfacial anisotropy and kinetic anisotropy. The role of these two anisotropies is often confused. In this paper we intend to clarify its role by giving several theorems which can be proved by development of the theory of very singular diffusion equations.

We consider an anisotropic curvature flow equations for an evolving (hyper)surface $\left\{\Gamma_{t}\right\}_{t \geqslant 0}$ (physically a crystal surface) in $\mathbf{R}^{n}(n \geqslant 2)$ of the form

$$
V=M(\vec{n})\left(\kappa_{\gamma}+\sigma\right) \text { on } \Gamma_{t},
$$

where $V$ denotes the normal velocity of $\Gamma_{t}$ in the direction of unit normal $\vec{n}$ of $\Gamma_{t}$. The function $M$ is called a mobility. It is a positive function defined on the unit sphere. In many models it is considered 
as a given function. The quantity $1 / M$ is called a kinetic coefficient [38]. The quantity $\kappa_{\gamma}$ is a weighted mean curvature or anisotropic mean curvature. It is the first variation of the interfacial energy $I$ of the hypersurface $\Gamma$ :

$$
I(\Gamma)=\int_{\Gamma} \gamma_{0}(\vec{n}) \mathrm{d} \mathcal{H}^{n-1},
$$

where $\gamma_{0}$ is a given positive function depending on the normal (orientation) called the interfacial energy density and $\mathrm{d} \mathcal{H}^{n-1}$ denotes the area element. We may write

$$
\kappa_{\gamma}=-\frac{\delta I}{\delta \Gamma}
$$

in a symbolic way. Its explicit form is formally as

$$
\kappa_{\gamma}=-\operatorname{div}_{\Gamma}\left[\left(\nabla_{p} \gamma\right)(\vec{n})\right] \quad \text { on } \quad \Gamma,
$$

where $\gamma(p)=|p| \gamma_{0}(p /|p|)$ is the 1-homogeneous extension of $\gamma_{0}$ to $\mathbf{R}^{n}$ and $\operatorname{div}_{\Gamma}$ is the surface divergence and $\nabla_{p} \gamma$ is the gradient of $\gamma$. If $\gamma_{0}$ is identically equal to 1 so that $\gamma_{0}$ is isotropic, the interfacial energy $I$ is nothing but the surface area of $\Gamma$. In this case $\gamma(p)=|p|$ and $\kappa_{\gamma}=-\operatorname{div}_{\Gamma} \vec{n}$ which is $(n-1)$-times mean curvature. The function $\gamma_{0}$ is determined by a crystal lattice structure. At least in very low temperature it is theoretically computable [46], [39], [45]. It seems that there is no reasonable way to calculate mobility $M$. The anisotropy $\gamma_{0}$ is called interfacial anisotropy while the anisotropy in $M$ is called kinetic anisotropy. If both $\gamma_{0}$ and $M$ are isotropic, i.e. $\gamma_{0}$ and $M$ are constants and $\sigma=0$, then (1.1) is the mean curvature flow equation. The function $\sigma$ in (1.1) is a driving force. For a snow crystal growth it is supersaturation of water molecules on $\Gamma_{t}$. It may depend on the position and the time. In general model like the Stefan model it is coupled with diffusion equations outside crystal surface $\Gamma_{t}$. If $\sigma$ is given, the model (1.1) is called an interface controlled model (or local model).

To see the structure of (1.1) it is convenient to recall the notion of the Frank diagram [38]

$$
\text { Frank } \gamma=\{p \mid \gamma(p) \leqslant 1\} .
$$

If Frank $\gamma$ is convex, then (1.1) is at least degenerate parabolic under suitable regularity of $\gamma$, say $C^{2}$. If Frank $\gamma$ is convex but loses $C^{1}$ regularity, then (1.1) becomes a very singular diffusion equation and is nontrivial to handle. A typical example is the case when Frank $\gamma$ is a convex polytope. In this case $\gamma_{0}$ is called a crystalline energy density and (1.1) is called a crystalline flow. Formally, the polar set of Frank $\gamma$ called a Wulff shape of $\gamma$ of the form

$$
W_{\gamma}=\bigcap_{|\vec{m}|=1}\{x \mid x \cdot \vec{m} \leqslant \gamma(\vec{m})\}
$$

plays a role of a sphere in the sense that

$$
\kappa_{\gamma}=-(n-1) \quad \text { on } \quad \partial W_{\gamma}
$$

where $\vec{n}$ is taken outward. If Frank $\gamma$ has a corner, then $W_{\gamma}$ has a flat portion (a facet) with normal corresponding to the corner direction. To understand (1.6) in a reasonable way, one should interpret that the curvature $\kappa_{\gamma}$ on the facet of $\partial W_{\gamma}$ is not zero but some positive quantity despite the fact that 
the surface is flat. This suggests that $\kappa_{\gamma}$ is not an infinitesimal quantity. It should be defined in a nonlocal way. This nonlocal character causes several difficulties. For an evolving curve (i.e. $n=2$ ) various well-posedness results are established for the initial value problem for (1.1) when $\sigma$ is a spatially constant [18], [22].

However, for $n \geqslant 3$ or non-constant $\sigma$ even well-posedness is not well-established. The main reason for $n \geqslant 3$ is that $\kappa_{\gamma}$ may not be constant on a facet [8] (even if $\sigma=0$ ) if one interprets $\kappa_{\gamma}$ in a reasonable way. Similarly, for non-constant $\sigma$ the quantity $\kappa_{\gamma}+\sigma$ may not be constant on a facet [19].

For $n \geqslant 3$ the unique solvability result is established for $V=\gamma \kappa_{\gamma}$ for convex initial data [6] by a variational approach. When $\Gamma_{t}(n=2)$ is the graph of a function of one space variable, i.e. a graphlike function, the unique solvability result is established in [19], when the equation is written in a divergence form even if $\sigma$ may not be a spatially constant. Recently, a viscosity approach enables us to assert well-posedness for more general equations for graph-like functions [25] when $n=2$. This formulation allows "facet bending" or "facet breaking". An explicit solution allowing facet bending is constructed in various settings, e.g. [34], [29]. For a general background of these problems the reader is referred to reviews articles [26], [27], [23] and [5].

In this paper we consider (1.1) in a very simple setting. We consider a planar motion (i.e., $n=2$ ) and postulate that $\gamma_{0}$ is crystalline and $\sigma$ is a given positive constant. We start with a convex crystal surrounded by $\Gamma_{0}$ and show that facets are instantaneously formed. Such a kind of result is already proved for a different setting in [18] and recently studied for a case when Frank $\gamma$ has a curved part by P. Mucha [50] and P. Mucha and P. Rybka [51], [52] but for graphs. We call this phenomenon the instant formation of a facet. We next study whether or not a crystal becomes fully faceted after some short time. We only discuss a simple situation when Frank $\gamma$ is a regular polygon centered at the origin. If $M$ has the same symmetry as $\gamma_{0}$, a growing convex crystal starting from nearly the "critical size" (i.e. the curve satisfying $\kappa_{\gamma}+\sigma=0$ ) becomes fully faceted in a finite time and it is similar (homothetic) to $W_{\gamma}$. In this paper we prove these statements by approximating by a crystalline flow which is a system of ordinary differential equations [58], [1]. The approximation is justified by [22]. Note that even solvability is nontrivial for (1.1) for general (convex) initial data $\Gamma_{0}$ and it is established in [22]. The merit of crystalline approximation is that one can prove these statements as we intuitively observed. A fully faceted crystal grows further and its large time asymptotic is once influenced by the mobility. In fact, it is known that $\Gamma_{t} / t \rightarrow \sigma W_{M}$ (Hausdorff distance sense) as $t \rightarrow \infty$ [41]. So a fully faceted crystal may be rounded again. The whole time growth behavior starting from very small convex shape is numerically calculated by [44].

It is often believed that growth phenomena is due to mobility and that anisotropy of curvature is negligible. This is true for a scaled down profile of larger time asymptotics. However, if Frank $\gamma$ is $C^{2}$ and strictly convex so that the curvature $\kappa_{\gamma}$ has no nonlocal nature, there is no facet provided that $M$ is Lipschitz (continuous) by the strong maximum principle as observed in [44]. Unless the interfacial energy is degenerate or singular, it is impossible to explain formation of facets when $M$ is Lipschitz.

To explain snow crystal growth one has to couple (1.1) with a diffusion equation for $\sigma$ to form the Stefan type problem. One of reasonable ways to model snow crystal growth is to consider a quasi-static approximation of one-phase Stefan type problem with Gibbs-Thomson effect and kinetic cooling. Its explicit form is

$$
\begin{aligned}
& \Delta \sigma=0 \quad \text { in } \quad \Omega_{t} \subset \mathbf{R}^{3}, \\
& V=\partial \sigma / \partial \vec{n} \quad \text { on } \Gamma_{t}=\partial \Omega_{t} \text {, }
\end{aligned}
$$




$$
V=M(\vec{n})\left(\kappa_{\gamma}+\sigma\right) \quad \text { on } \quad \Gamma_{t},
$$

where $\Omega_{t}$ is a region outside crystal. Of course one should assign the value $\sigma$ at $|x|=\infty$ and initial surface $\Gamma_{0}$. (We take all physical constants just one for simplicity.) Recently, J. Barrett, H. Garcke and R. Nürnberg [2], [3], [4] invented a nice numerical scheme to calculate for singular $\gamma$ and reproduce snow crystals both for $n=2$ and $n=3$. They take realistic values for mobility due to experiments while basic physics (1.7)-(1.9) including interfacial energy is taken as discussed in physical literature [47]. In [4] it appears that they successfully reproduce Nakaya's diagram [53] on snow crystal shapes depending on temperature and supersaturation. In particular, they reproduce a hexagonal prism like shape when the crystal is very small. There is another approach based on cell automaton which also produce nice pictures of snow flakes in computation [37]; however, it is not clear in what way physical parameters like $\sigma_{\infty}$ is involved.

Mathematical analysis is not well-developed for (1.7)-(1.9) (called also one-phase Hele-Shaw type problem), even if Frank $\gamma$ is smooth and strictly convex. If the problem has two-phases, it is known that the initial value problem is locally solvable and equilibrium shape is stable [14] when $\gamma$ is isotropic. The anisotropic version has been established in [10]. For the one-phase problem the solvability is proved in [49] for isotropic case. If the equation (1.7) is replaced by the heat equation, the system is called the Stefan type problem and there is a large literature for two-phase problems; the reader is referred to [55] and papers cited there. However, except [43] one-phase problem is less studied.

If the interfacial energy is singular so that its Wulff shape is a cylinder, it is known that the initial value problem is locally solvable provided that one restricts the class of a solution as a cylindrical evolution [32]. In some cases there even exist self-similar solutions [33]. However, a facet may break if $\sigma$ is coupled so that $\sigma$ is not a constant [33]. It is restrictive to consider a class of cylindrical evolution. A sufficient condition is given so that a cylindrical evolution is still a "reasonable solution" and this is actually fulfilled for small self-similar solution [33].

In [60] evolution of snowflakes is calculated by (1.7)-(1.9); see also [62]. However, the term $\kappa_{\gamma}$ is omitted. According to [4], if there is no $\kappa_{\gamma}$, no hexagonal pattern is produced. It seems that the numerical treatment in [60] and [62] includes some "regularizing effect" corresponding to $\kappa_{\gamma}$ to reproduce snowflakes. Roughly speaking, anisotropy of mobility plays a role to determine rough shape of crystals (see also [61]) while anisotropy of interface energy plays a role to determine detailed shape.

Our results correspond to the case when the crystal is sufficiently small. Although for a snow crystal growth $\sigma$ is of course not a constant and also coupled, full faceted nature seems to be very similar. In fact, if the crystal is very small compared with gradient of $\sigma$, flatness (of a facet) is preserved; see e.g. [33]. In our analysis, when $\gamma_{0}$ is crystalline with $M>0$ and constant $\sigma>0$, the mobility $M$ plays a little role if one is interested in formation of facets. Even for general mobility full faceted nature itself does not seem to depend on mobility. Only singular interfacial energy plays a role. However, mobility in the direction of facet seems to be important to determine the "shape" of fully faceted crystal $\Gamma_{t}$. We do not touch these problems in this paper. However, if we discuss the shape of small slowly growing crystals, this point seems to be very important because there are several nice experiments [16], [48] which are believed to find equilibrium shape i.e. $\kappa_{\gamma}+\sigma=0$ but one should be careful about the role of mobility if $M$ is not proportional to $\gamma$.

This paper is organized as follows. In Section 2 we state our main result on instant formation of facet after reviewing definition and a few properties of solutions. In Section 3 we state our main result on formation of a fully faceted shape. In Section 4 we prove our main results by using a crystalline algorithm. Our selection of references is not at all exhaustive. 


\section{Instant formation of facet}

We first recall well-posedness results in [22] for (1.1) with $n=2$ when Frank $\gamma$ has singularities. Our formulation is based on adjustment of a level-set method developed by [14], [9]; see also [28]. Instead of considering (1.1), we consider

$$
u_{t} /|\nabla u|=M(\vec{n})\left(-\operatorname{div}\left(\nabla_{p} \gamma(\vec{n})\right)+\sigma\right) \quad \text { with } \quad \vec{n}=-\nabla u /|\nabla u|
$$

which is formally equivalent to (1.1) on $\Gamma_{t}=\left\{x \in \mathbf{R}^{2} \mid u(x, t)=0\right\}$. We consider (2.1) not only on $\Gamma_{t}$ but also for all $\mathbf{R}^{2} \times[0,+\infty)$. As in [28] we say that an open set $D \subset \mathbf{R}^{2} \times[0, \infty)$ is an open evolution of (1.1) with initial data $D_{0}$ if there is a solution $u \in C\left(\mathbf{R}^{2} \times[0, \infty)\right)$ of (2.1) such that $D=\{(x, t) \mid u(x, t)>0\}$ and $D_{0}=\{x \mid u(x, 0)>0\}$ such that $u$ equals a negative constant outside a big ball (depending on $t$ ). A closed evolution $E \subset \mathbf{R}^{2} \times[0, \infty)$ of (1.1) with initial data $E_{0}$ is defined in a parallel way by replacing $>$ by $\geqslant$. The set $\Gamma=E \backslash D$ is called an interface evolution which is a generalized solution of (1.1) starting with initial curve $\Gamma_{0}=E_{0} \backslash D_{0}$ with $E_{0}=\bar{D}_{0}$. Here by a solution of (2.1) we mean a certain viscosity like solution [22], which is a generalized notion of solution based on the maximum principle [28]. By definition the orientation $\vec{n}$ is taken outward from $D$. Our formulation restricts that $D(t)$ (resp. $E(t))$ the cross-section of $D$ (resp. $E$ ) at $t$, i.e. $D(t)=\left\{x \in \mathbf{R}^{2} \mid(x, t) \in D\right\}$ is bounded (resp. $\left.E(t)=\left\{x \in \mathbf{R}^{2} \mid(x, t) \in E\right\}\right)$. Moreover, for a given $T>0$ there is a big ball $B$, such that $D(t) \subset E(t) \subset B$ for all $t \in(0, T]$. We warn the reader that $E$ may be strictly larger than $\bar{D}$ for $E_{0}=\bar{D}_{0}$ even for smooth $\gamma$. This phenomenon is called fattening [14], [28]. Thus we say that $E$ is regular if $E=\bar{D}$ for $E_{0}=\bar{D}_{0}$. This implicitly assumes that there is no spike for $E_{0}$. by

We consider a class of singular interfacial energy including a crystalline energy. Let $\mathcal{I}$ be defined

$$
\begin{aligned}
\mathcal{I}=\left\{\gamma: \mathbf{R}^{2} \rightarrow\right. & (0, \infty) \mid \gamma \text { is convex, positively homogeneous of degree } 1 \\
& \text { and Frank } \gamma \text { is } C^{2} \text { except finitely many points. Moreover, }
\end{aligned}
$$

the curvature of $\partial$ (Frank $\gamma)$ is bounded except singularities $\}$.

Here is a typical well-posedness result proved in [22], where more general curvature flow equations are discussed.

Lemma 2.1 (Unique existence, [GG01, Corollary 8.2, Theorem 6.4]) Let $M$ be a nonnegative continuous function defined on $S^{1}=\left\{p \in \mathbf{R}^{2}|| p \mid=1\right\}$ and $\gamma \in \mathcal{I}$. Assume that $\sigma \in \mathbf{R}$ is a constant. For a given bounded open set $D_{0}$ (resp. closed set $E_{0}$ ) there is a unique open evolution $D$ (resp. closed evolution $E$ ) with initial data $D_{0}$ (resp. $R_{0}$ ) for (1.1).

It turns out such evolutions have a nice stability result [20]. So one can approximate the singular problem by a problem with smoother interfacial energy. Here is a typical result proved in [22].

LEmma 2.2 (Approximation, [GG01, Corollary 8.3]) Assume that a sequence of continuous functions $M_{\varepsilon}: S^{1} \rightarrow[0, \infty)$ converges to $M$ uniformly as $\varepsilon \rightarrow 0$ and that $\gamma_{\varepsilon} \in \mathcal{I}$ converges to $\gamma \in \mathcal{I}$ uniformly on $S^{1}$ as $\varepsilon \rightarrow 0$. Moreover, $\sigma_{\varepsilon} \in \mathbf{R} \rightarrow \sigma \in \mathbf{R}$. Let $E_{0}^{\varepsilon}$ and $E_{0}$ be bounded closed sets in $\mathbf{R}^{2}$. Let $E^{\varepsilon}$ be a closed evolution of

$$
V=M_{\varepsilon}(\vec{n})\left(\kappa_{\gamma_{\varepsilon}}+\sigma_{\varepsilon}\right)
$$


with initial data $E_{0}^{\varepsilon}$ and $E$ be a closed evolution of (1.1) with initial data $E_{0}$. Assume that

$$
d_{H}\left(E_{0}^{\varepsilon}, E_{0}\right) \rightarrow 0 \quad \text { as } \quad \varepsilon \rightarrow 0,
$$

where $d_{H}$ is the Hausdorff distance in $\mathbf{R}^{2}$. Assume that $E$ is regular. Then

(i) $d_{H}^{\prime}\left(E^{\varepsilon}, E\right) \rightarrow 0$ as $\varepsilon \rightarrow 0$, where $d_{H}^{\prime}$ denotes the Hausdorff distance in $\mathbf{R}^{2} \times[0, T]$ for a fixed $T>0$.

(ii) Assume that $t \mapsto E(t)$ is continuous as a set-valued function for $t \geqslant 0$. Assume that $E$ is strongly regular in $[0, T]$ in the sense that $E(t)=\overline{D(t)}$ for all $t \in[0, T]$, where $D$ is the open evolution of (1.1) with initial data $D_{0}$ such that $E_{0}=\overline{D_{0}}$. Then

$$
\lim _{\varepsilon \rightarrow 0} \sup _{0 \leqslant t \leqslant T} d_{H}\left(E^{\varepsilon}(t), E(t)\right)=0 .
$$

Approximation by smoother energy is first established in [15] for a graph-like function for a special equations of divergence type. It is extended for general equations by [20]. Note that our approximation result (Lemma 2.2) also allows the approximation of the problem with smooth $\gamma$ by singular $\gamma_{\varepsilon}$ like crystalline. Such a kind of approximations is discussed in a special setting graph-like solutions by [15], [36] and convex curves [35].

We now consider a convex initial data. We would like to show that the convexity is preserved and the corresponding closed evolution is regular.

THEOREM 2.3 Assume the same hypothesis of Lemma 2.1 concerning $M, \gamma$ and $\sigma$. Let $E_{0}$ be the closure of a bounded, open and convex set $D_{0}$. Let $E$ be the closed evolution of (1.1) with initial data $E_{0}$.

(i) (Convexity preserving) The cross-section $E(t) \subset \mathbf{R}^{2}$ is convex for all $t \geqslant 0$.

(ii) (Non-fattening) The closed evolution $E$ is regular. Moreover, $E$ is strongly regular in $[0, T]$ for all $T<T_{*}$, i.e. $E(t)=\overline{D(t)}$ for $0 \leqslant t<T_{*}$, where $D$ is the open evolution of (1.1) with initial data $D_{0}$ and $T_{*}$ is the extinction time, i.e. $T_{*}=\sup \{t \mid$ int $E(t) \neq \varnothing\} \leqslant \infty$.

Proof. For smooth energy $\gamma$ we have convexity preserving property proved by [30]; see [28, Remark 3.5.5]. For a level-set equation (2.1) we have concavity preserving property for an auxiliary function $u(x, t)$ for (2.1) for smooth $\gamma$ [28, Remark 3.5.2]. Note that in our definition an auxiliary function is taken so that it equals a negative constant at the space infinity. However, this restriction is not essential. In fact, we may allow spatially uniformly continuous functions. Thus at least for convex set $E_{0}$ there is a solution $u \in C\left(\mathbf{R}^{2} \times[0, \infty)\right)$ of $(2.1)$ in $\mathbf{R}^{2} \times(0, \infty)$ such that $x \mapsto u(x, t)$ is concave and uniformly continuous with the property:

$$
E=\{(x, t) \mid u(x, t) \geqslant 0\}, \quad E(t)=\{x \mid u(x, t) \geqslant 0\} .
$$

By convergence to a singular problem [22, Theorem 8.1] such $u$ converges to the problem with singular interfacial energy if it is approximated by smooth interfacial energy. Thus for singular $\gamma$ the concavity preserving property $u$ for (2.1) is inherited. This in particular implies that the convexity of $E(t)$ is preserved and also yields non-fattening property since the level-set flow is a level set of a concave function.

We are now in position to state our main result on the instant formation of facet. 
THEOREM 2.4 (Instant formation of facet) Assume that Frank $\gamma$ is a convex polygon, i.e. $\gamma \in \mathcal{I}$ is crystalline. Assume that $M: S^{1} \rightarrow(0, \infty)$ is continuous and $\sigma \in \mathbf{R}$. Let $E_{0}$ be the closure of a bounded open and convex set $D_{0}$. Let $E$ be the closed evolution of (1.1) with initial data $E_{0}$. Let $\left\{\Gamma_{t}\right\}$ be the interface evolution defined by $\Gamma_{t}=E(t) \backslash D(t)$. Let $\mathcal{N} \subset S^{1}$ be the singular set of $\gamma$, i.e. $\gamma_{0}=\left.\gamma\right|_{S^{1}} \in C^{2}\left(S^{1} \backslash \mathcal{N}\right)$ and $\left.\nabla \gamma\right|_{S^{1}}$ has jumps exactly on $\mathcal{N}$. Then, for each $\vec{n} \in \mathcal{N}$ there is a facet (a flat portion) of $\Gamma_{t}$ (the boundary of a convex open set) with orientation $\vec{n}$ for all $t \in\left(0, T_{*}\right)$, where $T_{*}$ is the extinction time. In other words, a facet is instantaneously created and stays.

We shall postpone the proof to Section 4 . The basic idea is to approximate by crystalline algorithm. This is possible by a general approximation result (Lemma 2.2) and consistency result in [21]. It seems to be possible to extend (Theorem 2.4) to a general $\gamma \in \mathcal{I}$ not necessarily crystalline but we do not pursue this problem.

Our Theorem 2.4 implicitly asserts that the solution becomes "essentially admissible" even if initially it is a non-essentially admissible crystal. For essentially admissibility, see Section 4. A missing direction in $\mathcal{N}$ is actually formed. Such a problem is studied in [24] and [54] for a polygonal evolution.

\section{Formation of fully faceted small crystals}

We consider the equation (1.1) when $\sigma$ is a positive constant. It is easy to see that the rescaled Wulff shape $C=(1 / \sigma) W_{\gamma}$ is a stationary closed evolution of (1.1). By the comparison principle [22] if the initial data int $E_{0}$ includes $C$, the closed evolution $E$ of (1.1) with initial data always includes $C$ and never disappears for all $t>0$. If $E_{0} \subset$ int $C$, then the corresponding solution $E$ disappears in finite time if $\inf M>0$. Thus the set $C$ is called a critical shape. We are interested in shapes of crystals $E(t)$ when $E_{0}$ includes in $C$ but close to $C$. It turns out that if we assume that Frank $\gamma$ is a convex polygon so that its Wulff shape $W_{\gamma}$ is a dual polygon, then $E(t)$ becomes a convex polygon with the same set of orientations as that of $W_{\gamma}$ after some time. We call this polygon a fully faceted shape with respect to $\gamma$. To avoid pathological situation we impose a kind of monotonicity of $M$ in a singular direction $\vec{n} \in \mathcal{N}$ of $\gamma$.

We extend $M$ to $\mathbf{R}^{2}$ so that it is 1-homogeneous, i.e.,

$$
M(p)=|p| M(p /|p|), \quad p \neq 0 .
$$

The Frank diagram is defined like (1.4), i.e.,

$$
\text { Frank } M=\left\{p \in \mathbf{R}^{2} \mid M(p) \leqslant 1\right\} .
$$

Let $C\left(\mathbf{m}_{1}, \mathbf{m}_{2}\right)$ be a closed (proper) cone in $\mathbf{R}^{2}$ spanned by two unit vectors $\mathbf{m}_{1}, \mathbf{m}_{2}$. Let $f_{i}$ be the height function of $W_{i}=$ Frank $M \cap C\left(\vec{n}_{i-1}, \vec{n}_{i+1}\right)$ in a direction $\vec{n}_{i} \in \mathcal{N}$ as in [22]. Here $\vec{n}_{i-1}$ and $\vec{n}_{i+1}$ are adjacent directions of $\vec{n}_{i}$ in Frank $M$. In other words,

$$
\begin{gathered}
f_{i}(x)=\sup \left\{y \mid x \vec{n}_{i}^{\perp}+y \vec{n}_{i} \in W_{i}\right\}, \\
x \in \operatorname{dom} f_{i}=\left\{x \mid x \vec{n}_{i}^{\perp}+y \vec{n}_{i} \in W_{i} \text { for some } y \in \mathbf{R}\right\} .
\end{gathered}
$$

Here $\vec{n}_{i}^{\perp}$ is an unit vector orthogonal to $\vec{n}_{i}$. (To fix idea we take $\vec{n}_{i}^{\perp}$ so that $\vec{n}_{i}^{\perp} \cdot \vec{n}_{i+1}>0$.) 


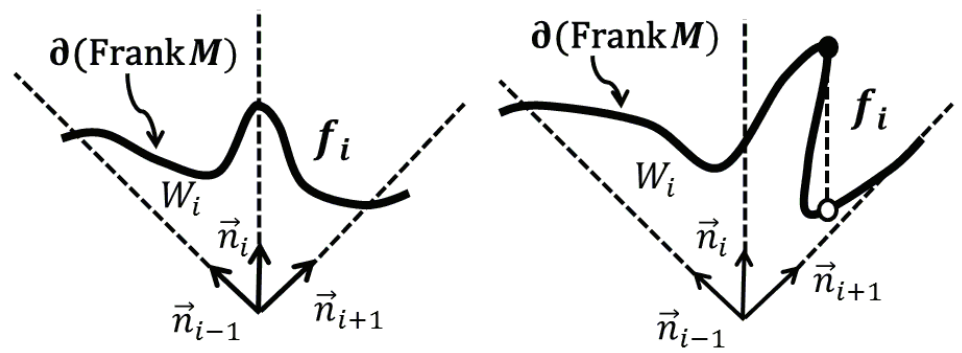

FIG. 1. Height functions of $W_{i}$

DEFINITION 3.1 (Monotonicity of $M$ ) We say that $M$ is monotone with respect to $\mathcal{N}$ if for each $\vec{n}_{i} \in \mathcal{N}$ there is a constant $\alpha_{i} \in(0, \pi / 2)$ such that

$$
\begin{array}{ll}
f_{i}(x+h)-f_{i}(x) \geqslant-\left(\tan \alpha_{i}\right) h, & h>0, x>0, x+h \in \operatorname{dom} f_{i}, \\
f_{i}(x-h)-f_{i}(x) \geqslant-\left(\tan \alpha_{i}\right) h, & h>0, x<0, x-h \in \operatorname{dom} f_{i},
\end{array}
$$

where $f_{i}$ is the height function of $W_{i}$ in the direction of $\vec{n}_{i}$.

The left in Figure 1 is an example that $M$ is monotone in the direction of $\vec{n}_{i}$ while the right in Figure 1 is an example that $M$ is not monotone.

We give a typical result for a fully faceted phenomena.

THEOREM 3.2 (Fully faceted phenomena) Assume that Frank $\gamma$ is a regular $k$-polygon centered at the origin. Assume that $M: S^{1} \rightarrow(0, \infty)$ is continuous and $\sigma>0$. Assume furthermore that $M$ is monotone with respect to $\mathcal{N}$, where $\mathcal{N}$ is the singular set of $\gamma$. Let $E_{0}$ be the closure of a bounded open and convex set $D_{0}$ containing the critical shape $C=(1 / \sigma) W_{\gamma}$. Let $E$ be the interface evolution of (1.1) with initial data $E_{0}$.

(a) If $E_{0}$ is sufficiently close to $C$, there $E(t)$ becomes fully faceted with respect to $\gamma$ in finite time $t=t_{0}$ with some $t_{0}>0$.

(b) Assume that $E_{0}$ is sufficiently close to $C$ so that $E$ has the property in (a). If $E_{0}$ and $M$ has the same symmetry as $\gamma$ i.e., $\mathbf{Z}_{k}$-symmetry in the sense that $E_{0}$ is invariant under rotation of angle $2 \pi / k$, then the full faceted shape is similar to $W_{\gamma}$ on some time interval i.e. $E(t)=c(t) W_{\gamma}$ for $t \in\left(t_{0}, t_{1}\right)$ with some $t_{0}, t_{1}>0$ with some positive constant $c(t)$.

(c) Assume that $E_{0}$ is sufficiently close to $C$ so that $E$ has the property in (a). If $E_{0}$ has the same symmetry as $\gamma$ and if $M$ is proportional to $\gamma$, then $E(t)=c(t) W_{\gamma}$ for all $t>t_{0}$ with some $t_{0}>0$ with some positive constant $c(t)$.

REMARK 3.3 (i) Unless $M$ is proportional to $\gamma$, the large time asymptotics may be different from $W_{\gamma}$ up to dilation even in case (b). Actually, it is essentially known that $E(t) / t \rightarrow W_{M}$ (Hausdorff distance sense) so $t \rightarrow \infty$, where $W_{M}$ is the Wulff shape of $M$. This asymptotic behavior is proved in [41] for smooth $\gamma$ but it can be easily extended to the singular case as noted in [44].

(ii) There are several numerical simulations for $E$ by changing anisotropy of $\gamma$ and $M$ in [44]. In their examples for a short time a fully faceted shape with respect $\gamma$ is obtained. In a large time 
the "rough shape" is $t W_{M}$ but facets with orientation $\vec{n} \in \mathcal{N}$ stay for all time even if $W_{M}$ and $W_{\gamma}$ has different orientations of their edges.

(iii) So far the effect of the mobility seems to be little for $E(t)$ which is close to $C$. However, if $M / \gamma$ depends on $\vec{n} \in \mathcal{N}$, then the fully faceted shape is not similar $W_{\gamma}$ (nor $W_{M}$ ). We conjecture that $\sigma E(t)$ converges to $c W_{\tilde{\gamma}}$ with $\tilde{\gamma} \kappa_{\tilde{\gamma}}=M \kappa_{\gamma}$ as $\sigma \rightarrow 0$ with some constant $c>0$. Such $\tilde{\gamma}$ is very related to the existence problem for self-similar shrinking solutions for $V=M \kappa_{\gamma}$. In fact, there is a unique self-similar solution whose cross section is similar to $W_{\tilde{\gamma}}$ for some $\tilde{\gamma}$ provided that $\gamma$ and $M$ is smooth and $\gamma(\vec{n})=\gamma(-\vec{n}), M(\vec{n})=M(-\vec{n})$ and that Frank $\gamma$ is strictly convex; see [17], [11], [12] and a review paper [26] and a book [28]. A similar result is proved for crystalline flow by [56], [57]. So it is likely that the value of $M(\vec{n}) / \gamma(-\vec{n})$ on $\mathcal{N}$ plays an important role to determine the fully faceted shape. For example in three dimension the Wulff shape $W_{\gamma}$ is a hexagonal prolonged prism while we often find thin or thick hexagonal prism of snowflakes. This is caused by a difference of $M / \gamma$ on basal and prism surface.

(iv) Even if $\sigma$ is not constant, admissible facets stay admissible provided that the crystal is sufficiently small (so that $\kappa_{\gamma}$ is large) with respect to the gradient of $\sigma$. On the facet the driving force turns to be equal to its average over the facet in this case [19], [33]. In fact, a comparison principle holds for such small crystals [7]. Moreover, even one considers a coupled system (1.7)-(1.9), there exists a special solution which is self-similar and fully faceted for a certain time when $M$ and $\gamma$ is chosen so that $M / \gamma$ is constant as in the case of (c). This is actually proved for special $\gamma$ and $M$ in [33].

(v) The monotonicity of $M$ with respect to $\gamma$ is not very restrictive. Typical examples include $M \equiv 1$ (for $k>4$ ) and $M \equiv \gamma$. Examples in numerical simulation [44], [4] also fulfill this monotonicity.

\section{Approximation by crystalline flow}

We shall prove Theorems 2.4 and 3.2 by approximating by (generalized) crystalline flows. We first recall flows with no curvature term to explain corner preserving conditions.

\subsection{Corner preservation}

We consider the Hamilton-Jacobi equation

$$
V=M(\vec{n}) \sigma
$$

where $M>0$ is continuous and $\sigma$ is a positive constant. In other words, we drop the curvature term in (1.1). We consider the closure of convex polygonal domain $K_{0}$ with facets whose orientations consist of $\mathcal{M}=\left\{\mathbf{m}_{j}\right\}_{j=1}^{r} \subset S^{1}(r \geqslant 3)$. The numbering is taken clockwise. We consider a flow of (4.1) starting from $S_{0}=\partial K_{0}$. We are interested in the problem whether or not the solution stays a convex polygon with the same orientation of facets. For this purpose we recall an elementary geometric fact.

LEMMA 4.1 Let $H(\mathbf{m}, h)$ be the half space with orientation $\mathbf{m} \in S^{1}$ and the support parameter $h>0$, i.e.,

$$
H(\mathbf{m}, h)=\left\{x \in \mathbf{R}^{2} \mid x \cdot \mathbf{m} \leqslant h\right\} .
$$


Let $\mathbf{m}_{1}, \mathbf{m}_{2} \in S^{1}\left(\mathbf{m}_{1} \neq \mathbf{m}_{2}\right)$ satisfy $\mathbf{m}_{1} \cdot \mathbf{m}_{2}>0$. Let $\mathbf{n} \in S^{1}$ be a point on the (shorter) $\operatorname{arc} \overparen{\mathbf{m}_{1} \mathbf{m}_{2}}$. Let $h_{1}, h_{2}$ be positive parameters. Then

$$
H\left(\mathbf{m}_{1}, h_{1}\right) \cap H\left(\mathbf{m}_{2}, h_{2}\right) \subset H(\mathbf{n}, h)
$$

if and only if

$$
h \geqslant \frac{1}{\sin \varphi}\left(h_{1} \sin \varphi_{2}+h_{2} \sin \varphi_{1}\right) .
$$

Here $\varphi$ is the angle of (vectors) $\mathbf{m}_{1}$ and $\mathbf{m}_{2}$ and $\varphi_{\ell}$ is the angle of $\mathbf{m}_{\ell}$ and $\mathbf{n}$ so that $\varphi_{1}+\varphi_{2}=\varphi$. The equality in (4.2) holds if and only if $\partial H(\mathbf{n}, h) \cap H\left(\mathbf{m}_{1}, h_{1}\right) \cap H\left(\mathbf{m}_{2}, h_{2}\right)$ is a singleton.

We now consider the initial value problem for (4.1) with initial data $S_{0}=\partial K_{0}$. We may assume that $K_{0}$ includes the origin as an interior point so that it is of the form

$$
K_{0}=\bigcap_{j=1}^{r} H\left(\mathbf{m}_{j}, h_{j}^{0}\right) .
$$

The problem is whether or not

$$
\begin{gathered}
K_{t}=\bigcap_{j=1}^{r} H\left(\mathbf{m}_{j}, h_{j}(t)\right), \quad t>0 \\
h_{j}(t)=h_{j}^{0}+M\left(\mathbf{m}_{j}\right) \sigma t
\end{gathered}
$$

is the solution (the closed evolutions) of (4.1) starting from $K_{0}$ in level-set sense or viscosity sense (e.g. [28]). For example, if $M \equiv 1$, then the corner of solutions is immediately rounded. The next property easily follows from Lemma 4.1 and definition of solution.

Lemma 4.2 (Corner preservation) Assume that for all $j \in\{1, \ldots, r\} M$ satisfies

$$
M(\mathbf{m})=\frac{1}{\sin \varphi_{j j+1}}\left\{M\left(\mathbf{m}_{j}\right) \sin \varphi_{j+1}+M\left(\mathbf{m}_{j+1}\right) \sin \varphi_{j}\right\},
$$

where $\mathbf{m}$ is on the (shorter) arc between $\mathbf{m}_{j}$ and $\mathbf{m}_{j+1}$ and $\varphi_{j j+1}$ is the angle of $\mathbf{m}_{j}$ and $\mathbf{m}_{j+1}$ and $\varphi_{k}$ is the angle of $\mathbf{m}_{k}$ and $\mathbf{m}$ with convention that $\mathbf{m}_{r+1}=\mathbf{m}_{1}$. Then $K_{t}$ given by (4.4) is the solution of (4.1) (with $\sigma>0$ ) starting with $K_{0}$ given by (4.3).

REMARK 4.3 Actually, it is enough to assume $\geqslant$ in (4.5). The condition (4.5) is a necessary and sufficient condition so that the solution stays a polygon with orientations contained in $\mathcal{M}$ if $K_{0}$, which is not necessarily convex, has $\mathcal{M}$ as the set of orientations. We also note that some corner may disappear so Lemma 4.2 gives a condition that no new corners or round parts are created. The same condition (4.5) at each corner is given for example in [21, (3.2)] or [38] in a different way of description.

We give a geometric interpretation. We extend $M$ to $\mathbf{R}^{2}$ so that it is 1-homogeneous, i.e.,

$$
M(p)=|p| M(p /|p|), \quad p \neq 0 .
$$

The Frank diagram is defined like (1.4). 
Lemma 4.4 Assume that the polygonal domain whose vertices consist of points in $\mathcal{M}$ contains the origin. The condition (4.5) holds for all $j \in\{1, \ldots, r\}$ if and only if Frank $M$ is a polygon (not necessarily convex) whose vertices consist of $\left(1 / M\left(\mathbf{m}_{j}\right)\right) \mathbf{m}_{j}, j \in\{1, \ldots, r\}$ (allowing that the angle of a vertex equals $\pi$ ).

The proof is elementary so is left to the readers. If (4.5) is fulfilled for all $j \in\{1, \ldots, r\}$, then the orientation of the Wulff shape $W_{M}$ corresponding to direction of vertices of the convex hull of Frank $M$. In the solution $K_{t}$ determined (4.4) for sufficiently large $t$ the orientation which does not appear in $W_{M}$ stays bounded or disappear. The large time behavior in Remark 3.3 (i) can be directly proved in this setting.

\subsection{Crystalline flow for essentially admissible crystals}

We consider (1.1) for a polygonal flow. We first recall an essentially admissible evolving crystal which is a special evolving polygon preserved by a crystalline curvature flow equation. Let Frank $\gamma$ be a convex $k$-polygon. Let $q_{i}(i=1,2, \ldots, k)$ be its vertices. Then the singular set $\mathcal{N}\left(\subset S^{1}\right)$ is of the form

$$
\mathcal{N}=\left\{q_{i} /\left|q_{i}\right| \mid i=1,2, \ldots, k\right\} .
$$

We say that a simple oriented polygonal curve $S$ in $\mathbf{R}^{2}$ is an essentially admissible crystal if the (outward) unit normal vector $\mathbf{n}$ and $\hat{\mathbf{n}}$ of any adjacent segments (facets) of $S$ satisfies

$$
\frac{(1-\lambda) \mathbf{n}+\lambda \hat{\mathbf{n}}}{|(1-\lambda) \mathbf{n}+\lambda \hat{\mathbf{n}}|} \notin \mathcal{N}
$$

for any $\lambda \in(0,1)$. In other words, there is no singular direction between orientations $\mathbf{n}$ and $\hat{\mathbf{n}}$ of two adjacent segments. If we further impose that all orientations of facets of $S$ belongs to $\mathcal{N}$, then $S$ is called an admissible crystal which is a conventional admissibility proposed by [58], [1]; see also [31]. We say that a family of polygons $\left\{S_{t}\right\}_{t \in J}$ is an essentially admissible evolving crystal if $S_{t}$ is an essentially admissible crystal for all $t \in J$ and each corner moves continuously differentially in time as well as each facet keeps its orientation with $J=[0, T)$. The notion of essentially admissibility is found in [40], where $\sigma$ is assumed to be zero. An admissible evolving crystal is defined in a similar way.

We consider an essentially admissible crystal of closed polygonal curves with finite length for simplicity. By definition $S_{t}$ is of the form

$$
S_{t}=\bigcup_{j=1}^{r} S_{j}(t),
$$

where $S_{j}(t)$ is a maximal, nontrivial, closed segment and its unit outward normal vector is $\mathbf{n}_{j}$. Here we number facets clockwise. We say that $\left\{S_{t}\right\}_{t \in J}$ is a $\gamma$-regular flow [21] of (1.1) if

$$
V_{j}=M\left(\mathbf{n}_{j}\right)\left(\frac{\chi_{j} \Delta\left(\mathbf{n}_{j}\right)}{L_{j}(t)}+\sigma\right) \quad \text { on } \quad S_{j}(t), t \in J
$$

for $j=1,2, \ldots, r$, where $V_{j}$ denotes the normal velocity of $S_{j}(t)$ in the direction of $\mathbf{n}_{j}$. For the derivation, see e.g. [21]. The quantity $\chi_{j} \Delta\left(\mathbf{n}_{j}\right) / L_{j}(t)$ is a nonlocal weighted curvature $\kappa_{\gamma}\left(\mathbf{n}_{j}\right)$ for 
$\mathbf{n}_{j} \in \mathcal{N}$, where $L_{j}(t)$ denotes the length of $S_{j}(t)$. The quantity $\Delta\left(\mathbf{m}_{j}\right)$ is defined by

$$
\begin{array}{lll}
\Delta\left(\mathbf{m}_{j}\right)=\tilde{\gamma}^{\prime}\left(\theta_{j}+0\right)-\tilde{\gamma}^{\prime}\left(\theta_{j}-0\right) & \text { if } & \mathbf{m}_{j} \in \mathcal{N}, \\
\Delta\left(\mathbf{m}_{j}\right)=0 & \text { if } & \mathbf{m}_{j} \notin \mathcal{N},
\end{array}
$$

where $\mathbf{m}_{j}=\left(\cos \theta_{j}, \sin \theta_{j}\right)$ and $\tilde{\gamma}(\theta)=\gamma(\cos \theta, \sin \theta)$. The quantity $\chi_{j}$ is a transition number. It takes +1 (resp. -1 ) if $S_{t}$ is convex (resp. concave) in the direction of $\mathbf{n}_{j}$; we use the convention that $\chi_{j}=-1$ for all $j=1, \ldots, r$ if $S_{t}$ is convex. We note that $\Delta\left(\mathbf{n}_{i}\right)$ has a geometric meaning. It is the length of facet of the Wulff shape $W_{\gamma}$ with outward normal $\mathbf{n}_{i} \in \mathcal{N}$. We have assumed that $\sigma \in \mathbf{R}$ is a constant.

Since $\left\{S_{t}\right\}_{t \in J}$ is an essentially admissible evolving crystal, by elementary geometry one obtains a transport equation

$$
\dot{L}_{j}(t)=\frac{\mathrm{d} L_{j}(t)}{\mathrm{dt}}=\left(\cot \psi_{j}+\cot \psi_{j+1}\right) V_{j}-\frac{1}{\sin \psi_{j}} V_{j-1}-\frac{1}{\sin \psi_{j+1}} V_{j+1}
$$

for $j=1, \ldots, r$; index $j$ is considered modulo $r$. Here $\psi_{j}=\theta_{j}-\theta_{j-1}$ (modulo $\left.2 \pi\right)$ with $\mathbf{n}_{j}=$ $\left(\cos \theta_{j} \sin \theta_{j}\right.$ ). Thus the equation (4.7) forms a system of ordinary differentiation equations (ODE) with (4.8) for $L_{j}$ 's. A fundamental theory of ODE yields the (local-in-time) unique solvability of (4.7)-(4.8) for a given initial data $S_{0}$ for $J=\left[0, t_{0}\right)$, where $J$ is the maximal existence time interval. We shall restrict ourselves for convex $S_{0}$. Arguing in a similar way as in [21] and [42], one observes that only facet with $\mathbf{n}_{j} \notin \mathcal{N}$ may disappear for $\gamma$-regular flow at $t=t_{0}$ if the enclosed set of $S_{t_{0}-0}$ has no interior. One can extend the solution after $t_{0}$ by solving (4.7)-(4.8) if the enclosed set of $S_{t_{0}-0}$ has no interior. In the case when $\sigma=0$ we just repeat this procedure to continue the "solution" until the time $T_{0}$ when the enclosed area is zero. Such a solution is called a crystalline flow of (1.1) and $T_{0}$ is called an extinction time. Such a flow is well-studied in [59] for $\sigma=0$.

One should be careful to see the consistency of a crystalline flow with an interface evolution (also called a level-set flow). This topic is well-discussed for admissible evolving crystals in [21]. It is easy to adjust their argument for essentially admissible evolving crystals when $\sigma=0$ under corner preservation condition. However, if $\sigma>0$ some facets with non-admissible direction, i.e. $\mathbf{m}_{j} \notin \mathcal{N}$ need to be created to be a level-set flow when "admissible facet" becomes large. Because of the presence of $M$ a new facet may be created. We prepare some notion of convexification.

Definition 4.5 (Convexification associated with $\mathcal{Z}$ ) Let $C\left(\mathbf{m}_{1}, \mathbf{m}_{2}\right)$ be a closed (proper) cone spanned by two unit vectors $\mathbf{m}_{1}, \mathbf{m}_{2}$. Let $F$ be a closed set. Define

$$
F_{C\left(\mathbf{m}_{1}, \mathbf{m}_{2}\right)}=\operatorname{co}\left(F \cap C\left(\mathbf{m}_{1}, \mathbf{m}_{2}\right)\right),
$$

where co denotes the convexification. For $\mathcal{Z}=\left\{\mathbf{s}_{j}\right\}_{j=1}^{\ell} \subset S^{1}$ let

$$
\operatorname{co}(F ; \mathcal{Z})=\bigcup_{j=1}^{\ell} F_{C\left(\mathrm{~s}_{j}, \mathrm{~s}_{j+1}\right)}
$$

and call $\operatorname{co}(F ; \mathcal{Z})$ the convexification of $F$ associated with $\mathcal{Z}$. Here $\mathbf{s}_{j}$ is numbered clockwise with convention that $\mathbf{s}_{\ell+1}=\mathbf{s}_{1}$. Here the angle of $\mathbf{s}_{j}$ and $\mathbf{s}_{j+1}$ is assumed to be less than $\pi$ (so that $\ell \geqslant 3$ and the polygon whose vertices consist of all points of $\mathcal{Z}$ encloses the origin). 
Definition 4.6 Let $M$ be a continuous 1-homogeneous function in $\mathbf{R}^{2}$ such that $\left.M\right|_{S^{1}}>0$. For a given $\mathcal{Z} \subset S^{1}$ let $M_{\mathcal{Z}}$ be the 1-homogeneous function defined by

$$
\operatorname{Frank}\left(M_{\mathcal{Z}}\right)=\operatorname{co}(\operatorname{Frank} M ; \mathcal{Z})
$$

We call $M_{\mathcal{Z}}$ the hull of $M$ associated with $\mathcal{Z}$.

Assume that $M$ fulfills the corner preserving condition (4.5) with respect to a given set $\mathcal{M}=\left\{\mathbf{m}_{j}\right\}_{j=1}^{r}$. Then by Lemma 4.4 Frank $M$ is a polygon whose vertices consist of $1 /$ $M\left(\mathbf{m}_{j}\right) \mathbf{m}_{j}, j=1, \ldots, r$. In this case, evidently $M_{\mathcal{M}}=M$. For $\mathcal{N} \subset \mathcal{M}$ consider $M_{\mathcal{N}}$, the hull of $M$ associated with $\mathcal{N}$, i.e., $\operatorname{Frank}\left(M_{\mathcal{N}}\right)=\operatorname{co}(\operatorname{Frank} M, \mathcal{N})$. Let $\mathcal{M}_{\mathcal{N}}$ be the set of all vertex directions of Frank $\left(M_{\mathcal{N}}\right)$. By definition $\mathcal{M}_{\mathcal{N}} \subset \mathcal{M}$.

We give a generic condition which is especially preserved under evolution. In particular, we are interested in sufficient conditions so that no new facet is created. For this purpose we need to modify Frank $M$ depending upon the length of an admissible facet, i.e., a facet with the orientation in the singular set $\mathcal{N} \subset S^{1}$. Assume that $\sigma>0$. For a given $L_{i} \in[0, \infty]$ we set

$$
\Sigma_{i}=\left\{\lambda \mathbf{n}_{i} \mid 0 \leqslant \lambda \leqslant 1 /\left[M\left(\mathbf{n}_{i}\right)\left(1-\Delta\left(\mathbf{n}_{i}\right) / L_{i} \sigma\right)_{+}\right]\right\}, \quad \mathbf{n}_{i} \in \mathcal{N}
$$

with interpretation that

$$
\begin{aligned}
\Sigma_{i} & =\left\{\lambda \mathbf{n}_{i} \mid 0 \leqslant \lambda\right\} \quad \text { when } \quad L_{i} \sigma \leqslant \Delta\left(\mathbf{n}_{i}\right), \\
\Sigma_{i} & =\left\{\lambda \mathbf{n}_{i} \mid 0 \leqslant \lambda \leqslant 1 / M\left(\mathbf{n}_{i}\right)\right\} \quad \text { when } \quad L_{i}=\infty .
\end{aligned}
$$

We further denote the totality of these rays by $\Sigma(L)$, i.e.,

$$
\Sigma(L)=\bigcup_{i=1}^{k} \Sigma_{i}
$$

for $L=\left(L_{1}, \ldots, L_{k}\right)$.

Definition 4.7 (Property G) Let $\mathcal{M}$ be a finite set of $S^{1}$ containing $\mathcal{N}$. Assume that $M$ satisfies (4.5) with respect to $\mathcal{M}$. Assume that $\mathcal{K} \subset S^{1}$ satisfies $\mathcal{N} \subset \mathcal{K} \subset \mathcal{M}$. Assume that $\sigma>0$. For a given $L$ we say that $\mathcal{K}$ has the property $\mathrm{G}_{L}$ if all $\mathbf{m} / M(\mathbf{m})(\mathbf{m} \in \mathcal{M} \backslash \mathcal{K})$ belongs to the interior of the convexification of Frank $M \cup \Sigma(L)$ associated with $\mathcal{K}$, i.e. co (Frank $M \cup \Sigma(L), \mathcal{K})$. In the case $L=(\infty, \ldots \infty)$ by definition Frank $M=$ Frank $M \cup \Sigma(L)$ and we simply write $\mathrm{G}_{L}$ by $\mathrm{G}_{\infty}$. Let $K$ be the closure of a convex polygonal region in $\mathbf{R}^{2}$. Let $\mathcal{K}$ be the set of all orientations of facets of $K$ (satisfying $\mathcal{N} \subset \mathcal{K} \subset \mathcal{M}$ ). Let $L_{i}$ denote the length of admissible facet of $K$ whose orientation is $\mathbf{n}_{i} \in \mathcal{N}(1 \leqslant i \leqslant k)$. We say that $K$ has the property $\mathrm{G}$ if $\mathcal{K}$ has the property $\mathrm{G}_{L}$ with $L=\left(L_{1}, \ldots, L_{k}\right)$.

Evidently, the property $\mathrm{G}_{\infty}$ implies $\mathrm{G}_{L}$ so if $\mathcal{K}$ has the property $\mathrm{G}_{\infty}$, then $K$ has the property $\mathrm{G}$ independent of the length of an admissible facet.

To understand property $\mathrm{G}$ we consider the case when $\mathcal{N}$ is empty. We move a convex set $K_{0}$ by (4.1) with $\sigma>0$. More precisely, we consider the closed evolution of (4.1) starting from $K_{0}$. Assume that $\partial K_{0}$ is a convex polygon and the set of all orientations of facets of $K_{0}$ satisfies $\mathrm{G}_{\infty}$. It is easy to see that the property $\mathrm{G}$ is preserved although the set of orientations may decrease. No new facet is created. 
In the case when $\mathcal{N}$ is non-empty the closed evolution of (1.1) is more complicated when $\sigma>0$. Since $\mathrm{G}$ is an open property, during a short time, the property $\mathrm{G}$ is preserved. However, if the length of an admissible facet becomes large, there might be chance that some non-admissible facets with direction in $\mathcal{M} \backslash \mathcal{K}$ near the admissible direction, i.e. the direction $\mathbf{n}_{i} \in \mathcal{N}$ may be created so that it is consistent with the level-set flow. A general principle is that in a growing crystal a slow facet remains while a fast facet may disappear. The speed depends on the length for an admissible facet and this may break the property G. This kind of phenomena never occurs in the case $\sigma=0$.

We consider $\gamma$-regular flow of (1.1) starting from $S_{0}=\partial K_{0}$ and assume that the set $\mathcal{K}$ of orientations of $K_{0}$ agrees with $\mathcal{M}$. We consider the case $\sigma>0$. Evidently, $K_{0}$ has the property G. There is a chance that some non-admissible facets may disappear at some time $t_{0}$ but we extend $\gamma$ regular flow with initial data $S_{t_{0}-0}$ (enclosing $K_{t_{0}-0}$ ) as far as the property $\mathrm{G}$ is preserved. However, there might be chance that this extended $\gamma$-regular flow may lose the property $\mathrm{G}$ at some time $t_{1}$ so we need to modify by inserting some non-admissible facets.

Definition 4.8 (Modified $\gamma$-regular flow) Let $\mathcal{M}$ be a finite set of $S^{1}$ containing $\mathcal{N}$. Assume that $M$ satisfies (4.5) with respect to $\mathcal{M}$. Let $\left\{S_{t}\right\}_{t \in\left[0, t_{1}\right)}$ be an extended $\gamma$-regular flow of (1.1) (with $\sigma>0$ ) with initial data $S_{0}=\partial K_{0}$ and $S_{0}$ is a convex polygon such that the set of its orientation agrees with $\mathcal{K}(\mathcal{N} \subset \mathcal{K} \subset \mathcal{M})$. Assume that $K_{t}$ enclosed by $S_{t}$ has the property $\mathrm{G}$ for $t \in\left[0, t_{1}\right)$ but loses the property $\mathrm{G}$ at $t=t_{1}$. If there is a non-admissible direction $\mathbf{m}_{j} \in \mathcal{M} \backslash \mathcal{K}_{t_{1}}$ such that $\mathbf{m}_{j} / M\left(\mathbf{m}_{j}\right)$ is on the set

$$
\partial\left(\operatorname{co}\left(\text { Frank } M \cup \Sigma\left(L_{t_{1}}\right)\right), \mathcal{K}_{t_{1}}\right),
$$

then we insert all these non-admissible facets with such orientations for $t>t_{1}$ and solve (4.7) with adding these facets. Here $\mathcal{K}_{t}$ denotes the set of orientations of $K_{t}$ and $L_{t}=\left(L_{1, t}, \ldots, L_{k, t}\right)$ is the set of lengths of admissible facets of $K_{t}$. Note that some inserted facets may instantaneously disappear but the resulting solution for $t>t_{1}$ close to $t_{1}$ has the property G. We call this new solution modified $\gamma$-regular flow.

We say that an extended $\gamma$-regular flow of (1.1) starting from $S_{0}=\partial K_{0}$ is a (generalized) crystalline flow of (1.1) when $\sigma \leqslant 0$ provided that the set of orientation of $K_{0}$ equals $\mathcal{M}$. In the case $\sigma>0$ we call modified $\gamma$-regular flow of (1.1) starting from $S_{0}=\partial K_{0}$ (with $\mathcal{K}=\mathcal{M}$ ) a (generalized) crystalline flow of (1.1). For $\sigma>0$ a generalized crystalline flow preserves the property $\mathrm{G}$ except times of inserting non-admissible facets.

We are ready to state the consistency with the closed evolution, which develops no fattening as observed in Theorem 2.1.

Lemma 4.9 (Consistency) Assume the same hypotheses of Theorem 2.4 concerning $\gamma, M, \sigma$. Assume that $E_{0}$ is a convex closed polygonal region such that $S_{0}=\partial E_{0}\left(=\cup_{j=1}^{r} S_{j}(0)\right)$ is essentially admissible (with respect to $\gamma$ ). Let $E$ be the closed evolution of (1.1) with initial data $E_{0}$. Then $S_{t}=\partial E(t)$ is a (generalized) crystalline flow in $J=\left[0, T_{*}\right)$ with initial data $S_{0}$ provided that the corner preservation property (4.5) holds for all $j=1, \ldots, r$. Here $T_{*}$ is the extinction time $\leqslant \infty$.

The proof is similar to [21] but of course more involved. We take $\mathcal{M}$ consisting of all orientations of facets of the initial convex polygon $S_{0}$.

The criterion to insert non-admissible facet for $\sigma>0$ is based on the fact whether property $\mathrm{G}$ holds. As far as $\mathrm{G}$ holds, there need no creation of non-admissible facets. If an admissible faced becomes large, then its growing speed is larger and may lose the property G, so it may not prohibit 
creation of suitable non-admissible facets. Here is a condition for no creation of non-admissible facets. We invoke the monotonicity condition for $M$ given in Definition 4.6.

LEMmA 4.10 (No creation of non-admissible facets) Let $\mathcal{M}$ be a finite set of $S^{1}$ containing $\mathcal{N}$ and assume that $M$ satisfies (4.5) with respect to $\mathcal{M}$. Assume that $M$ is monotone with respect to $\mathcal{N}$ and with parameter $\alpha=\left(\alpha_{1}, \ldots, \alpha_{k}\right)$. Assume that $\mathcal{K}(\mathcal{N} \subset \mathcal{K} \subset \mathcal{M})$ has the property $\mathrm{G}_{0}$ with $\sigma>0$.

(i) There exists a constant $L_{i}^{*}>\Delta\left(\mathbf{n}_{i}\right) / \sigma$ such that if $L_{i} \leqslant L_{i}^{*}(i=1, \ldots, k)$ then $\mathcal{K}$ has the property $\mathrm{G}_{L}$ with $L=\left(L_{1}, \ldots, L_{k}\right)$. The constant $L_{i}^{*}$,s can be taken so that it only depends on $\alpha$ (independent of $\mathcal{K}$ ).

(ii) Let $K$ be a convex closed polygonal region where the set of its orientations equals $\mathcal{K}$. Then there is $\delta^{\prime}>0$ depending only on $\alpha$ such that if $K \subset C_{\delta^{\prime}}=\left(1+\delta^{\prime}\right) C, C=(1 / \sigma) W_{\gamma}$, then $K$ has the property $\mathrm{G}$.

The second statement is a simple collorary of the first statement because smaller $\delta$ we choose, $L_{i}$ 's becomes closer to $\Delta\left(\mathbf{n}_{i}\right) / \sigma$. The first statement is easily proved by elementary geometry.

Our generalized crystalline flow approximates the solution of (1.1) as we see below.

The corner preserving condition (4.5) uniquely interpolates the values of $M(\mathbf{m})$ from the value of $M$ at $\mathbf{m}_{j}(j=1, \ldots, r)$. The way of interpolation is given in [21, (3.3)]. Geometrically speaking, if $\sigma>0$, one considers an $r$-polygon $F$ whose vertices consist of $\mathbf{m}_{j} / M\left(\mathbf{m}_{j}\right)$. The interpolated $\tilde{M}$ is defined such that Frank $\tilde{M}$ agrees with $F$.

Notice that if the set $\left\{\mathbf{m}_{j}\right\}_{j=1}^{r(\varepsilon)}$ converges to $S^{1}$ in the sense of Hausdorff distance as $\varepsilon \rightarrow 0$, $\tilde{M}=\tilde{M}^{\varepsilon}$ converges to $M$ uniformly on $S^{1}$. Thus, combining Lemma 2.2 and Theorem 2.3 with consistency (Lemma 4.9), we are able to conclude the convergence of crystalline algorithm.

LEMMA 4.11 (Convergence of crystalline algorithm) Assume the same hypotheses of Theorem 2.4 concerning $\gamma, M, \sigma$. Let $E_{0}$ be the closure of bounded, convex open set $D_{0} \neq \emptyset$. Let $E$ be the closed evolution of (1.1) with initial data $E_{0}$ and let $\left\{\Gamma_{t}\right\}_{t \geqslant 0}$ be the corresponding level-set flow with initial data $\Gamma_{0}=E_{0} \backslash D_{0}$ with extinction time $T_{*} \in(0, \infty]$. Let $E_{0}^{\varepsilon}$ be a convex polygon such that its boundary $S_{0}^{\varepsilon}=\cup_{j=1}^{r(\varepsilon)} S_{j}^{\varepsilon}(0)$ is an essentially admissible crystal (with respect to $\gamma$ ). Let $S_{t}^{\varepsilon}$ be the crystalline flow with initial data $S_{0}^{\varepsilon}$. Then

$$
\sup _{0 \leqslant t \leqslant T} d_{H}\left(S_{t}^{\varepsilon}, \Gamma_{t}\right) \rightarrow 0
$$

for all $T \in\left(0, T_{*}\right)$ provided that $d_{H}\left(S_{0}^{\varepsilon}, \Gamma_{0}\right) \rightarrow 0$ as $\varepsilon \downarrow 0$.

There are several ways to approximate $\Gamma_{0}$ by an essentially admissible crystal $S_{0}^{\varepsilon}$. We here give a typical way. A convex set $E_{0}=\overline{D_{0}}$ is written by using a suitable support function $h(\mathbf{m})$ (convex in $\mathbf{R}^{2}$ ) so that

$$
E_{0}=\left\{x \in \mathbf{R}^{2} \mid x \cdot \mathbf{m} \leqslant h(\mathbf{m}) \text { for all } \mathbf{m} \in S^{1}\right\} .
$$

We approximate $E_{0}$ by $E_{0}^{\varepsilon}$ by

$$
E_{0}^{\varepsilon}=\left\{x \in \mathbf{R}^{2} \mid x \cdot \mathbf{m} \leqslant h(\mathbf{m}) \text { for all } \mathbf{m} \in \mathcal{N} \cup \mathcal{M}_{\varepsilon}\right\} .
$$

where $\mathcal{M}_{\varepsilon}=\left\{\left(\cos \theta_{i}, \sin \theta_{i}\right) \mid \theta_{i+1} \leqslant \theta_{i}\right.$ with $\left.\left|\theta_{i+1}-\theta_{i}\right| \leqslant \varepsilon, i=1,2, \ldots N(\varepsilon)\right\}$. It is not difficult to see that $E_{0}^{\varepsilon} \rightarrow E_{0}$ in the Hausdorff distance sense. Evidently, $\partial E_{0}^{\varepsilon}$ is an essentially admissible crystal by modifying $h$ slightly if necessary. 
Because of this approximability, approximation by crystalline flow is often called a crystalline algorithm [36], [35] since crystalline flow is obtained by solving ODEs. The convergence rate is proved for heat equation and curve shortening equation of graph-like function for smooth solution in [36] and for convex curve [35]. Note that convergence result is also proved in [15] for graphs. However, in both cases there is no driving force $\sigma$.

\subsection{Formation of admissible facets}

We use above approximation to conclude instant formation of an admissible facets.

Proof of Theorem 2.4. We use crystalline approximation given in Lemma 4.11. Since $E^{\varepsilon}(t)$ and $E(t)$ are convex sets we may represent these sets by

$$
E^{\varepsilon}(t)=\bigcap_{j=1}^{r(\varepsilon)}\left\{x \in \mathbf{R}^{2} \mid x \cdot \mathbf{n}_{j} \leqslant d_{j}^{\varepsilon}(t)\right\}
$$

with $d_{j}^{\varepsilon}(t)$ 's, we say $d_{j}^{\varepsilon}$ is he support function at $\mathbf{n}_{j}$. For the case $\sigma>0$ we have to allow that $d_{j}^{\varepsilon}(t)$ may have jump for non-admissible facet since some non-admissible facets may be created. By definition of $T_{*}$ for $T \in\left(0, T_{*}\right)$ there is a small open disk $B$ such that $B \subset E(t)$ and $B \subset E^{\varepsilon}(t)$ for $t \in[0, T]$ and for small $\varepsilon>0$. We may assume that the center of $B$ is the origin. This implies

$$
\inf _{0<\varepsilon<\varepsilon_{0}} \inf _{t \in[0, T]} d_{j}^{\varepsilon}(t)>0 \quad \text { for } j=1, \ldots, r
$$

We shall prove that the length of a facet with the orientation $\mathbf{n}_{\ell} \in \mathcal{N}$ of $\Gamma_{t}$ is positive in a dense subset of $(0, T)$. Let $L_{\ell}(t)$ be the length of the facet with normal $\mathbf{n}_{\ell}$ allowing that $L_{\ell}(t)=0$, i.e., the facet is degenerated to a point. We do not assume that $L_{\ell}(0)>0$. By the convergence of convex set and Lemma 4.11 we conclude that $L_{l}^{\varepsilon}(t) \rightarrow L_{\ell}(t)$ and $d_{l}^{\varepsilon}(t) \rightarrow d_{\ell}(t)$ uniformly on $[0, T]$, where $d_{\ell}(t)$ is the support function at $\mathbf{n}_{\ell} \in \mathcal{N}$ corresponding to the facet of $\Gamma_{t}$. Assume that $L_{\ell}(t)=0$ for $\left[t_{0}, t_{1}\right] \subset(0, T)$. Since $\dot{d}_{l}^{\varepsilon}(t)$ is the normal velocity $V_{\ell}=V_{\ell}^{\varepsilon}$, integrating (4.7) over $\left[t_{0}, t_{1}\right]$ yields

$$
d_{l}^{\varepsilon}\left(t_{1}\right)-d_{l}^{\varepsilon}\left(t_{0}\right)=M\left(\mathbf{n}_{\ell}\right) \int_{t_{0}}^{t_{1}}\left(\frac{-\Delta\left(\mathbf{n}_{\ell}\right)}{L_{\ell}^{\varepsilon}(t)}+\sigma\right) \mathrm{d} t, \mathbf{n}_{\ell} \in \mathcal{N}
$$

The left hand side is bounded from below as $\varepsilon \rightarrow 0$. However, this is impossible since $L_{l}^{\varepsilon}(t) \rightarrow 0$ uniformly in $\left[t_{0}, t_{1}\right]$. We have thus proved that $L_{\ell}(t)>0$ for a dense subset of $(0, T)$. Since $\mathcal{N}$ is finite, there is a dense set $J_{0}$ of $(0, T)$ such that $L_{\ell}(t)>0$ for all $t \in J_{0}$ and $\mathbf{n}_{\ell} \in \mathcal{N}$.

As for the proof of crystalline flow [42], [21] combining (4.7) and (4.8) for approximation, one is able to prove that facets of $\Gamma_{t}$ with orientation in $\mathcal{N}$ has a positive length for a short time if it has initially of positive length. If admissible facets surrounded by parts of non-admissible direction, these parts move by (4.1) so it does not affect admissible facets especially when admissible facets are short. We do not give a detailed proof. This implies $L_{\ell}(t)>0$ for all $t \in(0, T]$ so the proof is now complete.

\subsection{Fully faceted pattern}

We shall prove formation of a fully faceted pattern stated in Theorem 3.2. The basic idea is to show a similar statement for crystalline flows and a general flow by approximation by a crystalline flow. Of 
course, one should be careful to check that the time duration of fully faceted pattern is take uniform with respect to approximation.

We shall always assume that

Frank $\gamma$ is a regular $k$-polygon centered at the origin,

to simplify the situation. Our strategy to prove formation of a fully faceted pattern consists of (i) upper bound for growth of admissible facets by a self-similar solution and (ii) lower bound for growth of non-admissible facets. Two bounds imply disappearance of non-admissible facets in some time interval by Lemma 4.1 .

Let $C$ be the critical shape, i.e., $C=(1 / \sigma) W_{\gamma}$. We consider

$$
V=a\left(\kappa_{\gamma}+\sigma\right)
$$

with $a=\sup _{S^{1}} M$. As well-known, the equation (4.10) admits a self-similar crystalline flow of the form $S_{t}^{*}=z(t) \partial C$ under (4.9) $[38,12 \mathrm{G}]$. The function $z(t)$ solves

$$
\dot{z}(t)=a b^{-1}(-1 / z(t)+\sigma)
$$

where $b=\gamma\left(\mathbf{n}_{i}\right)$. If initial data is taken as a boundary of $C_{\delta}=(1+\delta) C$ with $\delta>0$, the corresponding growing self-similar solution $S_{t}^{*}$ encloses convex set $K_{t}^{*}$ of the form

$$
K_{t}^{*}=\bigcap_{i=1}^{k} H\left(\mathbf{n}_{i}, \lambda(t)\right), \lambda(t)=z(t) b, z(0)=(1+\delta) / \sigma, \mathbf{n}_{i} \in \mathcal{N} .
$$

Proposition 4.12 (Upper bound) Assume (4.9) and $\sigma>0$. Let $\Gamma_{t}^{*}$ be the crystalline flow of (1.1) of the form

$$
\Gamma_{t}^{*}=\partial G_{t}^{*}, \quad G_{t}^{*}=\bigcap_{i=1}^{k} H\left(\mathbf{n}_{i}, d_{i}^{*}(t)\right), \quad t \in[0, T]
$$

starting from $\Gamma_{0}^{*}=\partial C_{\delta}$. Then $G_{t}^{*} \subset K_{t}^{*}$ or equivalently

$$
d_{i}^{*}(t) \leqslant \lambda(t)
$$

where $\lambda(t)=z(t) b$ is given by (4.11) with $z(0)=(1+\delta) / \sigma$.

Proof. We first note that the (outward) normal velocity of each facet of $\Gamma_{t}^{*}$ is always non-negative. Indeed, $G_{0}^{*}=C_{\delta} \subset G_{h}^{*}$ for small $h>0$ because speed of $\Gamma_{0}^{*}$ is positive everywhere. By a comparison principle [31] $G_{t}^{*} \subset G_{t+h}^{*}$ for all $t>0$ which yields that the normal velocity of $\Gamma_{t}^{*}$ is non-negative.

We have observed that $\kappa_{\gamma}+\sigma \geqslant 0$ on each facet of $\Gamma_{t}^{*}$. Thus we conclude that $\Gamma_{t}^{*}$ is a "subsolution" of (4.10). By a comparison principle [31] we see that $G_{t}^{*} \subset K_{t}^{*}$.

Proposition 4.13 (Upper bound for admissible facets) Assume (4.9) and $\sigma>0$. Let $\mathcal{M} \subset S^{1}$ so that $\mathcal{N} \subset \mathcal{M}$ where $\mathcal{N}$ is the set of admissible directions. Let $K_{0}$ be a closed convex polygonal region satisfying the property G. Assume that $C \subset K_{0} \subset C_{\delta}$. Let $\partial K_{t}$ be the (generalized) crystalline flow starting from $\partial K_{0}$. Then $K_{t} \subset K_{t}^{*}$. In particular,

$$
h_{i}(t) \leqslant \lambda(t) \quad \text { for } \quad \mathbf{n}_{i} \in \mathcal{N}, \quad t \in[0, T]
$$

where $\lambda(t)$ is the same as Proposition 4.12 provided that $K_{t}$ is expressed as (4.4) for $t \in[0, T]$. 
Proof. This follows from comparison principle of a level-set flow [22]. One should interpret (generalized) crystalline flow as a level-set flow of (1.1) by interpolating $M$ outside $\mathcal{M}$ so that (4.5) holds by Lemma 4.9. The comparison principle yields $K_{t} \subset G_{t}^{*}$ so that Proposition 4.12 yields the desired result.

Proposition 4.14 (Lower bound for non-admissible facets) Assume the same hypothesis of Proposition 4.13. Then

$$
h_{j}(t) \geqslant m \sigma t+h_{j}(0) \quad \text { for } \quad \mathbf{m}_{j} \in \mathcal{M} \backslash \mathcal{N}, \quad t \in[0, T]
$$

with $m=\inf _{S^{1}} M$ provided that a non-admissible facet of orientation $\mathbf{m}_{j}$ exists in $[0, T]$.

Proof. For non-admissible facet we know

$$
\dot{h}_{j}(t)=M\left(\mathbf{m}_{j}\right) \sigma \geqslant m \sigma
$$

which yields the desired result.

Proof of Theorem 3.2. We first prove (a) for a generalized crystalline flow. We take $\delta^{\prime}$ as in Lemma 4.10. For a given $T>0$ we take $\delta>0$ sufficiently small, say $\delta<\delta_{0}$ so that

$$
\lambda^{\delta}(t) \leqslant \frac{\left(1+\delta^{\prime}\right)}{\sigma} b \quad \text { in } \quad[0, T]
$$

with $\lambda^{\delta}(t)=z(t) b$, where $z$ be a solution of (4.11) with $z(0)=(1+\delta) / \sigma$. (This is possible since $\lambda^{\delta}$ converges to a constant $b / \sigma$ uniformly on $[0, T]$ as $\delta \rightarrow 0$.) If $K_{0} \subset C_{\delta}$, then the solution $K_{t}$ is contained in $K_{t}^{*}$ by Proposition 4.13. Thus $K_{t} \subset C_{\delta^{\prime}}$ for $t \in[0, T]$. By Lemma $4.10 K_{t}$ has the property $\mathrm{G}$ for all $t \in[0, T]$ which means that no new facets are created so we may assume that $K_{t}$ is expressed as (4.4) as in Proposition 4.13 on the time interval $[0, T]$.

We take $T$ large, say $m \sigma T>\bar{c}\left(1+\delta^{\prime}\right) b / \sigma$, where $\bar{c}=1 / \cos (\varphi / 2), \varphi=2 \pi / k$. Evidently, $\lambda^{\delta}$ is a convex, monotone increasing function and converges to a constant $b / \sigma$ uniformly on $[0, T]$ as $\delta \rightarrow 0$. By this behavior of $\lambda^{\delta}$ there is always an interval $\left[t_{1}^{\delta}, t_{2}^{\delta}\right) \subset(0, \infty)$ such that

$$
\bar{c} \lambda^{\delta}(t) \leqslant m \sigma t+b / \sigma \quad \text { on } \quad\left[t_{1}^{\delta}, t_{2}^{\delta}\right) \quad \text { with } \quad t_{1}^{\delta}<T
$$

with sufficiently small $\delta$, say $\delta<\delta_{1}\left(<\delta_{0}\right)$. (By the choice of $\delta_{0}$ and $T$ the inequality $t_{1}^{\delta}<T$ is guaranteed.)

We shall prove that a non-admissible facet with orientation $\mathbf{m}_{j}$ must disappear before $t=t_{1}^{\delta}$ if $\delta<\delta_{1}$. Assume that $\mathbf{m}_{j}$-facet exists up to $t \leqslant T$. There is $\mathbf{n}_{i}, \mathbf{n}_{i+1} \in \mathcal{N}$ such that $\mathbf{m}_{j}$ is between $\mathbf{n}_{i}$ and $\mathbf{n}_{i+1}$ on $S^{1}$. By Lemma 4.1

$$
h_{j}(t)<\frac{1}{\sin \varphi}\left(h_{i}(t) \sin \varphi_{1}+h_{i+1}(t) \sin \varphi_{2}\right)
$$

where $\varphi=2 \pi / k, \varphi_{1}+\varphi_{2}=\varphi, \varphi_{1}>0, \varphi_{2}>0$. By Proposition 4.13 we have $h_{i}(t) \leqslant \lambda^{\delta}(t)$ so that

$$
h_{j}(t)<\bar{c} \lambda^{\delta}(t)
$$


here $\bar{c}=\sup \left\{\left(\sin \varphi_{1}+\sin \varphi_{2}\right) / \sin \varphi \mid \varphi_{1}+\varphi_{2}=\varphi, \varphi_{1}>0, \varphi_{2}>0\right\}=1 / \cos (\varphi / 2)$. By geometry we have

$$
h_{j}(0) \geqslant b / \sigma \text {. }
$$

Proposition 4.14 now implies

$$
h_{j}(t) \geqslant m \sigma t+b / \sigma
$$

to get

$$
\bar{c} \lambda^{\delta}(t)>m \sigma t+b / \sigma
$$

which evidently contradicts (4.12). Thus all non-admissible facets disappear at some $t \leqslant t_{1}^{\delta}$ and is not created until time $T$.

Since we know that crystalline flow approximates a general level-set flow by Lemma 4.11, we get the same conclusion as above for general convex initial data by noting that all constants are independent of approximations. One should note that the constant appearing in the monotonicity of $M$ can be taken uniformly in the approximation of $M$. This implies that $t_{1}^{\delta}$ is taken independent of approximations. Thus the proof of (a) is now complete.

If we have symmetry, the symmetry is preserved by uniqueness of solution. This implies (b) since $\mathbf{Z}_{k}$-symmetric admissible polygon must be a regular $k$-polygon. In the case (c) since $k$-regular polygon always satisfies $\mathrm{G}$, there occurs no facet creation of non-admissible facets thus the solution stays as a $k$-regular polygon.

REMARK 4.15 In this section we consider a crystalline flow to (1.1) for admissible evolving convex crystals. Such a problem is studied when $\sigma=0$ in [59], where it is shown that all non-admissible facets disappear before the flow shrinks to a point. In the case $\sigma=0$, there is no need to insert non-admissible facets during evolutions so the problem is substantially different.

Acknowledgments. The authors are grateful to Professor Harald Garcke and Professor Etsuro Yokoyama for informative remarks. The authors are grateful to the anonymous referee for his/her careful reading. This work is partly supported by the Japan Society for the Promotion of Science through grant Kiban (S) 21224001, Kiban (A) 23244015, Houga 25610025.

\section{REFERENCES}

1. Angenent, S. B., \& Gurtin, M. E. Multiphase thermomechanics with interfacial structure. II. Evolution of an isothermal interface. Arch. Rational Mech. Anal. 108 (1989), 323-391. Zb10723.73017 MR1013461

2. Barrett, J. W., Garcke, H., \& NÜrnberg, R. On stable parametric finite element methods for the Stefan problem and the Mullins-Sekerka problem with applications to dendritic growth. J. Comput. Phys. 229 (2010), 6270-6299. Zbl1201.80075 MR2660305

3. Barrett, J. W., Garcke, H., \& Nürnberg, R. Finite element approximations of one-sided Stefan problems with anisotropic approximately crystalline, Gibbs-Thomson law. Adv. Differential Equations 18 (2013), 383-432. Zbl1271.80005 MR3060200

4. Barrett, J. W., GARCKe, H., \& NÜRnberG, R. Numerical computations of facetted pattern formation in snow crystal growth. Physical Review E 86 (2012), 011604, arXiv:1202.1272v1.

5. Bellettini, G. An introduction to anisotropic and crystalline mean curvature flow, Proceedings of minisemester on evolution of interfaces, Sapporo 2010. Hokkaido University Technical Report Series in Math. 145 (2010), 102-159. 
6. Bellettini, G., Caselles, V., Chambolle, A., \& Novaga, M. Crystalline mean curvature flow of convex sets. Arch. Ration. Mech. Anal. 179 (2006), 109-152. Zbl1148.53049 MR2208291

7. Bellettini, G., Goglione, R., \& Novaga, M. Approximation to driven motion by crystalline curvature in two dimensions. Adv. Math. Sci. Appl. 10 (2000), 467-493. Zb10979.53075 MR1769163

8. Bellettini, G., Novaga, M., \& Paolini, M. Facet-breaking for three-dimensional crystals evolving by mean curvature. Interfaces Free Bound. 1 (1999), 39-55. Zbl0934. 49023 MR1865105

9. Chen, Y. G., Giga, Y., \& Goto, S. Uniqueness and existence of viscosity solutions of generalized mean curvature flow equations. J. Differential Geom. 33 (1991), 749-786. Zbl0696. 35087 MR1100211

10. Deckelnick, K., \& Elliott, C. M. Local and global existence results for anisotropic Hele-Shaw flows. Proc. Roy. Soc. Edinburgh Sect. A 129 (1999), 265-294. Zbl0922.76150 MR1686701

11. Dohmen, C., \& Giga, Y. Selfsimilar shrinking curves for anisotropic curvature flow equations. Proc. Japan Acad. Ser. A Math. Sci. 70 (1994), 252-255. Zb10815. 34026

12. Dohmen, C., Giga, Y., \& Mizoguchi, N. Existence of selfsimilar shrinking curves for anisotropic curvature flow equations. Calc. Var. Partial Differential Equations 4 (1996), 103-119. Zb10847. 34042 MR1379195

13. Escher, J., \& Simonett, G. Classical solutions for Hele-Shaw models with surface tension. Adv. Differential Equations 2 (1997), 619-642. Zbl1023.35527 MR1441859

14. Evans, L. C., \& SPRUCK, J. Motion of level sets by mean curvature, I. J. Differential Geom. 33 (1991), 635-681. Zb10726.53029 MR1100206

15. FukUi, T., \& GigA, Y. Motion of a graph by nonsmooth weighted curvature. World Congress of Nonlinear Analysts '92, vol. I, Lakshmikantham, V. (ed.), 47-56, Walter de Gruyter, Berlin (1996). Zbl0860.35061 MR1389060

16. Furukawa, Y., \& Kohata, S. Temperature dependence of the growth form of negative crystal in an ice single crystal and evaporation kinetics for its surfaces. J. Crystal Growth 129 (1993), 571-581.

17. GAGE, M. E. Evolving plane curves by curvature in relative geometries. Duke Math. J. 72 (1993), 441466. Zb10798.53041 MR1248680

18. Giga, M.-H., \& GigA, Y. Evolving graphs by singular weighted curvature. Arch. Rational Mech. Anal. 141 (1998), 117-198. Zbl0896. 35069 MR1615520

19. GigA, M.-H., \& GigA, Y. A subdifferential interpretation of crystalline motion under nonuniform driving force. Dynamical systems and differential equations, Vol. I (Springfield, MO, 1996), Discrete Contin. Dynam. Systems 1998, Added Volume I, 276-287. MR1720610

20. GigA, M.-H., \& GigA, Y. Stability for evolving graphs by nonlocal weighted curvature. Comm. Partial Differential Equations 24 (1999), 109-184. Zb10924. 35177 MR1671993

21. GigA, M.-H., \& GigA, Y. Crystalline and level set flow - convergence of a crystalline algorithm for a general anisotropic curvature flow in the plane. GAKUTO Internat. Ser. Math. Sci. Appl. 13 (2000), 64-79. Zb10924.35177 MR1793023

22. GigA, M.-H., \& GigA, Y. Generalized motion by nonlocal curvature in the plane. Arch. Ration. Mech. Anal. 159 (2001), 295-333. Zbl1004. 35075 MR1860050

23. GigA, M.-H., \& GigA, Y. Very singular diffusion equations: second and fourth order problems. Jpn. J. Ind. Appl. Math. 27 (2010), 323-345. Zbl1213.35274 MR2746654

24. Giga, M.-H., Giga, Y., \& Hontani, H. Self-similar expanding solutions in a sector for a crystalline flow. SIAM J. Math. Anal. 37 (2005), 1207-1226. Zbl1093.74048 MR2192293

25. GigA, M.-H., GigA, Y., \& RYBKA, P. A comparison principle for singular diffusion equations with spatially inhomogeneous driving force for graphs. Hokkaido University Preprint Series in Math. 981 (2011). To appear in Arch. Ration. Mech. Anal.

26. GigA, Y. Anisotropic curvature effects in interface dynamics . Sūgaku 52 (2000), 113-117; English translation, Sügaku Expositions 16 (2003), 135-152. Zbl1247.53081 MR2019167

27. GIGA, Y. Singular diffusivity - facets, shocks and more. In: Applied math. entering the 21st century (eds. 
J. M. Hill and R. Moore), ICIAM (Sydney, 2003), SIAM (Philadelphia, 2004), 121-138. Zbl1144. 35300 MR2296265

28. GIGA, Y. Surface evolution equations - A level set approach. Birkhäuser Verlag, Basel (2006). Zbl1096. 53039 MR2238463

29. Giga, Y., Gorka, P., \& RYBKA, P. Evolution of regular bent rectangles by the driven crystalline curvature flow in the plane with a non-uniform forcing term. Adv. Differential Equations 18 (2013), 201242. Zbl06146745 MR3060195

30. Giga, Y., Goto, S., Ishit, H., \& SAto, M.-H. Comparison principle and convexity preserving properties for singular degenerate parabolic equations on unbounded domains. Indiana Univ. Math. J. 40 (1991), 443-470. Zbl0836.35009 MR1119185

31. Giga, Y., \& Gurtin, M. E. A comparison theorem for crystalline evolution in the plane. Quart. Appl. Math. 54 (1996), 727-737. Zb10862.35047 MR1417236

32. GigA, Y., \& RYBKA, P. Quasi-static evolution of 3-D crystals grown from supersaturated vapor. Differential Integral Equations 15 (2002), 1-15. Zbl1038. 35176 MR1869818

33. Giga, Y., \& RYBKA, P. Stability of facets of self-similar motion of a crystal. Adv. Differential Equations 10 (2005), 601-634. Zbl1109.35122 MR2133647

34. Giga, Y., \& RyB KA, P. Facet bending in the driven crystalline curvature flow in the plane. J. Geom. Anal. 18 (2008), 109-147. Zbl1146.53037 MR2365670

35. GIRÃO, P. M. Convergence of a crystalline algorithm for the motion of a simple closed convex curve by weighted curvature. SIAM J. Numer. Anal. 32 (1995), 886-899. Zb10830.65150 MR1335660

36. GirÃo, P. M., \& KoHn, R. V. Convergence of a crystalline algorithm for the heat equation in one dimension and for the motion of a graph by weighted curvature. Numer. Math. 67 (1994), 41-70. Zblo791. 65063 MR1258974

37. Gravner, J., \& Griffeath, D. Modeling snow crystal growth: A three-dimensional mesoscopic approach. Phys. Rev. E 79 (2009), 011601-1-18.

38. Gurtin, M. E. Thermomechanics of evolving phase boundaries in the plane. Clarendon Press (1993). Zbl0787.73004 MR1402243

39. Higuchi, K. On the shapes of ice crystals (in Japanese). Journ. Met. Soc. Japan 39 (1961), 237-248.

40. Hontani, H., Giga, M.-H., Giga, Y., \& DeGUCHI, K. Expanding selfsimilar solutions of a crystalline flow with applications to contour figure analysis. Discrete Appl. Math. 147 (2005), 265-285. Zbl1117. 65036 MR2127078

41. Ishit, H., Pires, G. E., \& Souganidis, P. E. Threshold dynamics type approximation schemes for propagating fronts. J. Math. Soc. Japan 51 (1999), 267-308. Zb10935. 53006 MR1674750

42. ISHIWATA, T. Motion of non-convex polygons by crystalline curvature and almost convexity phenomena. Japan J. Indust. Appl. Math. 25 (2008), 233-253. Zb11155. 53033 MR2431681

43. KNEISEL, C. Über das Stefan-Problem mit Oberflächspannung und thermischer Unterkühlung. Ph.D. thesis, Leibniz Universität Hannover, Germany (2007).

44. Kobayashi, R., \& Giga, Y. On anisotropy and curvature effects for growing crystals. Recent topics in mathematics moving toward science and engineering. Japan J. Indust. Appl. Math. 18 (2001), 207-230. Zb10981.35091 MR1842908

45. Kobayashi, T., \& Kuroda, T. Snow crystals, Chapter 10, Morphology of Crystals, edited by Sunagawa, I. Terra Scientific Publishing Company, Tokyo (1987), 645-743.

46. Krastanow, L. Über die Bildung und das Wachstum der Eiskristalle in der Atmosphäre. Met. Z. 60 (1943), 15-26.

47. Libbrecht, K. G. The physics of snow crystals. Rep. Prog. Phys. 68 (2005), 855-895.

48. Maruyama, M. Roughening transition of prism faces of ice crystals grown from melt under pressure. $J$. Crystal Growth 275 (2005), 598-605. 
49. Mucha, P. B. Stefan problem in a 2D case. Colloq. Math. 105 (2006), 149-165. Zbl1099.35179 MR2242506

50. MuchA, P. B. Regular solutions to a monodimensional model with discontinuous elliptic operator. Interfaces Free Bound. 14 (2012), 145-152. Zb106078133 MR2956321

51. Mucha, P. B., \& Ryb Ka, P. A note on a model system with sudden directional diffusion. J. Stat. Phys. 146 (2012), 975-988. Zbl1245.82020 MR2902450

52. MuchA, P. B., \& RYBKA, P. Well-posedness of sudden directional diffusion equations. http://arxiv . org/abs/1207.4929

53. NAKAYA, U. Snow Crystals: Natural and Artificial. Harvard University Press, Cambridge (1954).

54. OCHIAI, Y. Facet-creation between two facets moved by crystalline curvature. Master's thesis, University of Tokyo (2009).

55. Prüss, J., \& Simonett, G. Stability of equilibria for the Stefan problem with surface tension. SIAM J. Math. Anal. 40 (2008) 675-698. Zbl1157. 35502 MR2438781

56. StANCU, A. Uniqueness of self-similar solutions for a crystalline flow. Indiana Univ. Math. J. 45 (1996), 1157-1174. Zb10873.35034 MR1444481

57. Stancu, A. Asymptotic behavior of solutions to a crystalline flow. Hokkaido Math. J. 27 (1998), 303320. Zb10989.53042 MR1637988

58. TAYlor, J. E. Constructions and conjectures in crystalline nondifferential geometry. In: Differential Geometry (eds. B. Lawson and K. Tanenblat), Proceedings of the Conference on Differential Geometry (Rio de Janeiro, 1991), Pitman Monographs Surveys Pure Appl. Math. 52 Pitman (1991), 321-336. Zb10725.53011 MR1173051

59. YaZAKi, S. Motion of nonadmissible convex polygons by crystalline curvature. Publ. Res. Inst. Math. Sci., Kyoto Univ. 43 (2007), 155-170. Zbl1132.53036 MR2317117

60. Yokoyama, E. Formation of patterns during growth of snow crystals. J. Crystal Growth 128 (1993), 251-257.

61. Yokoyama, E., Giga, Y., \& RYBKA, P. A microscopic time scale approximation to the behavior of the local slope on the faceted surface under a nonuniformity in supersaturation. Phys. D 237 (2008), 2845-2855. Zb105506290 MR2514066

62. Yokoyama, E., \& Kuroda, T. Pattern formation in growth of snow crystals occurring in the surface kinetic process and the diffusion process. Physical Review A 41 (1990), 2038-2049. 